\title{
Measuring the electric dipole moment of the neutron: The cryoEDM experiment
}

\author{
C A Baker, S N Balashov, V Francis, K Green, M G D van der Grinten, P S laydjiev ${ }^{1}$, \\ S N Ivanov ${ }^{2}$, A Khazov ${ }^{3}$, M A H Tucker and D L Wark \\ Science and Technology Facilities Council, Rutherford Appleton Laboratory, Chilton, Didcot \\ OX11 OQX, UK.
}

A Davidson, J R Grozier, M Hardiman, P G Harris, J R Karamath, K Katsika, J M Pendlebury, S J M Peeters, D B Shiers, P N Smith, C M Townsley and I Wardell

University of Sussex, Falmer, Brighton BNI 9QH, UK.

\section{Clarke ${ }^{* 4}$, S Henry, H Kraus and M McCann ${ }^{5}$ \\ University of Oxford, Keble Road, Oxford OX1 3RH, UK.}

\section{P Geltenbort}

Institut Laue-Langevin, BP156, F-38042 Grenoble, France.

\author{
H Yoshiki \\ University of Kure, Japan. \\ ${ }^{1}$ on leave of absence from INRNE, Sofia, Bulgaria \\ ${ }^{2}$ on leave of absence from PNPI, St Petersburg, Russia \\ 3 joint with the University of Sussex, Falmer, Brighton BN1 9QH, UK \\ ${ }^{4}$ E-mail: c.clarke1@physics.ox.ac.uk \\ 5 joint with Rutherford Appleton Laboratory, Chilton, Didcot, OXON OX11 OQX, UK
}

\begin{abstract}
The cryoEDM experiment at the Institut Laue-Langevin in Grenoble will measure the electric dipole moment (EDM) of the neutron with unparalleled precision. A neutron EDM arises due to $\mathrm{CP}$ violation. The cryoEDM experiment is sensitive to levels of CP violation predicted by many "beyond the standard model" theories and the result will therefore constrain or support these theories. The current limit to the neutron EDM stands at $\left|d_{n}\right|<2.9 \times 10^{-26} e \mathrm{~cm}$ as measured with a room temperature experiment. By operating in superfluid helium below $0.9 \mathrm{~K}$ and collecting high densities of ultra cold neutrons, the cryoEDM experiment will improve on the existing limit or measure an EDM. High precision magnetometry is essential to reduce the systematic errors in the cryoEDM experiment originating from changes in the magnetic environment. We present the cryoEDM apparatus and technologies.
\end{abstract}

European Physical Society Europhysics Conference on High Energy Physics

July 16-22, 2009

Krakow, Poland

\footnotetext{
* Speaker.
} 


\section{Introduction}

Measurements of particle electric dipole moments have been the subject of increasingly sensitive experiments since 1951 [1]. A particle electric dipole moment (EDM) breaks parity and time reversal symmetries. Therefore, assuming CPT invariance, particle EDMs provide an indication of the level of $\mathrm{CP}$ violation across all sectors of particle physics. As yet undiscovered sources of $\mathrm{CP}$ violation will show up in an EDM.

The weak sector $\mathrm{CP}$ violation of the SM contributes $\sim 10^{-31}$ to $10^{-33} \mathrm{e} \mathrm{cm}$ to the neutron $\mathrm{EDM}(\mathrm{nEDM})$ [2]. If this is the only contribution, measuring the $\mathrm{nEDM}$ is beyond the reach of any current experiment. Extensions to the SM (for example supersymmetry and left-right symmetric models) predict a larger nEDM in the range $10^{-25}$ to $10^{-28} e \mathrm{~cm}$ [3]. The current upper limit of the nEDM is $2.9 \times 10^{-26} e \mathrm{~cm}$ [4]. This already constrains some supersymmetric models and CP violation in the strong sector of the SM.

The entire measurement in the cryoEDM experiment takes place below $0.9 \mathrm{~K}$. The nEDM is measured through an NMR process that looks for a shift in the neutron precession frequency when subjected to external electric and magnetic fields. Tight control and measurement of the magnetic environment is essential. Due to advantages gained from working in cryogenic conditions, the sensitivity is predicted to be of the order $10^{-28} e \mathrm{~cm}$ which allows for a nEDM discovery or a new upper limit to the value.

\section{Method}

A neutron precesses in a magnetic field at the Larmor frequency due to its magnetic dipole moment $\left(\mu_{n}\right)$. With an electric field present, in addition to the magnetic field, the precession frequency shifts. The difference in precession frequency when subjected to parallel and antiparallel electric and magnetic fields is directly proportional to the electric dipole moment $\left(d_{n}\right)$.

As $d_{n} \ll \mu_{n}$, we need sensitivity within a small fraction of the Larmor frequency. This is achieved using Ramsey's method of separated oscillating fields [5]. Two short RF pulses are applied separated by a long ( $\sim 300 \mathrm{~s})$ precession period. If the applied frequency matches the precession frequency, the resonance case is met and the neutron spin is completely flipped anti-parallel to the magnetic field. A difference in the precession frequency and the applied frequency will cause the RF and neutrons' precession to get increasingly out of step during the free precession resulting in a final spin state dependant on the phase difference. Looking for a shift in frequency when the direction of the electric field is reversed requires operating at a frequency near the resonance frequency where the final number of spin up neutrons is most sensitive to the phase difference.

\section{The experimental set-up}

The cryoEDM experiment (Fig. 1) is based at the Institut Laue-Langevin (ILL), Grenoble. It produces, stores and detects ultra-cold neutrons (UCN) within superfluid ${ }^{4} \mathrm{He}$. The apparatus includes two cooling towers: tower I supplies the helium for the production of UCN and tower II cools the magnetic shields. A circulating ${ }^{3} \mathrm{He}$ expansion system cools the UCN production volume to $\sim 0.7 \mathrm{~K}$. The electric field is generated by a Spellman HV supply and applied to the resonance 


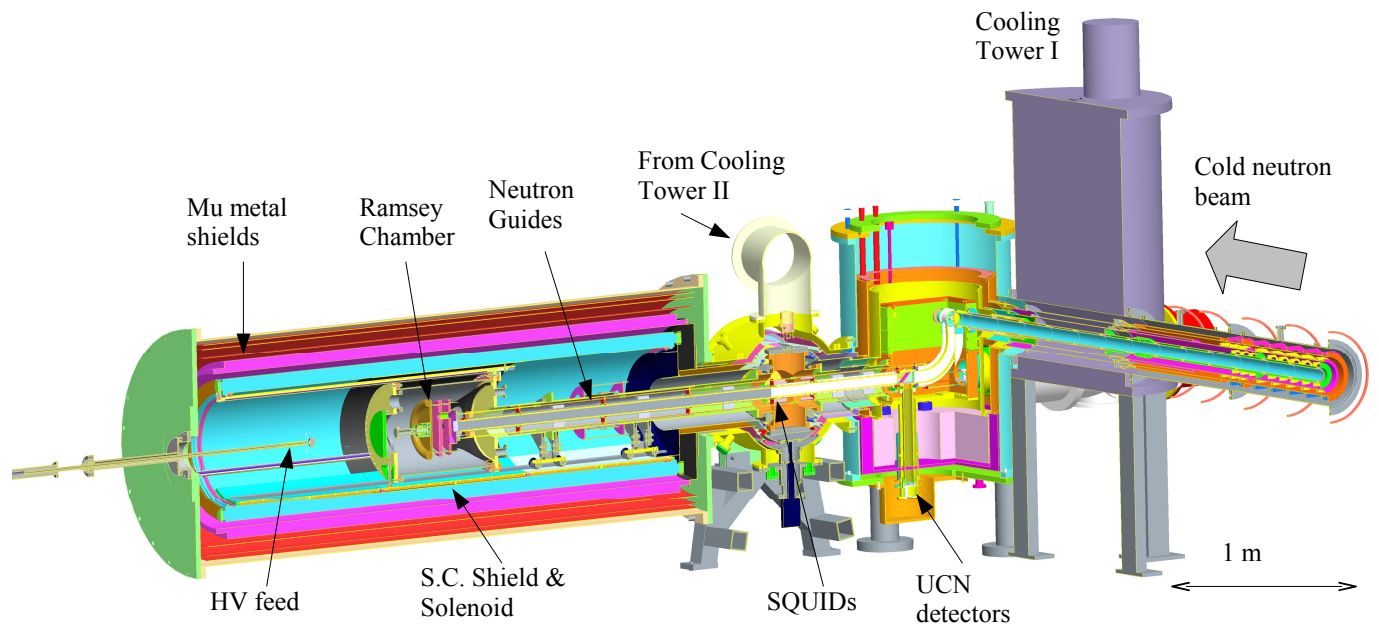

Figure 1: The components to the cryoEDM experiment.

cell (Ramsey Chamber). Not shown in the figure are the large pumps necessary for cryogenic operation.

\subsection{High density source of UCN}

The H53 cold neutron beam at ILL provides neutrons with energies $\sim 1 \mathrm{meV}$ which need to be cooled to $\sim 100 \mathrm{neV}$ in order to achieve long storage times for precession, increasing the sensitivity of the measurement. As the neutrons enter the experiment, they pass through a neutron polariser. UCN are produced by downscattering the cold neutrons in superfluid ${ }^{4} \mathrm{He}[6]$ which is produced in situ through superleaks [7]. The ${ }^{4} \mathrm{He}$ is very pure with less than 1 part in $10^{12}$ of ${ }^{3} \mathrm{He}$ which is a neutron absorber. In ${ }^{4} \mathrm{He}$, neutrons lose energy by phonon production. These UCN are transported from the UCN source to the resonance cells through beryllium-coated copper guides. The Fermi potential of beryllium is above the energy of the neutrons and so the guides are reflective. The neutrons are stored in cells made from beryllium oxide. There is a two cell design where one cell (termed the HV cell) has the electric field applied to it and the other cell is held neutral.

\subsection{Cryogenic Detectors}

After resonance, the neutrons are emptied from the resonance cells and counted by cryogenic solid state detectors [8]. They are silicon devices coated with lithium fluoride. Lithium reacts with neutrons to produce two charged products: an alpha and a triton particle. These products can then be detected by the silicon device producing a spectrum of two peaks for the two different particles. A layer of magnetically polarised iron on a detector reflects one spin polarisation so spin up neutrons can be counted separately from spin down.

\subsection{Magnetic control and monitoring}

In practice, a component of the difference in precession frequency will be from changing 
magnetic fields and not just $d_{n}$. To manage the effects of field fluctuations, the magnetic field needs to be stable and known well enough for corrections to be applied. A particular concern is from changes in the magnetic field that are correlated with reversals of the electric field as this could potentially produce a false nEDM signal.

Magnetic shielding comprising three layers of mu metal and one superconducting lead shield reduces the external magnetic field. The homogenous holding $B$ field of $5 \mu \mathrm{T}$ is produced by a superconducting solenoid and trim coils. To prevent local magnetic dipoles, all material near the resonance cells is non-magnetic.

Superconducting quantum interference devices (SQUIDs) are used to monitor the fields down to $0.1 \mathrm{pT}$ over the free precession time of $300 \mathrm{~s}$. They are located $\sim 2 \mathrm{~m}$ from the resonance cells so they do not introduce additional magnetic fields. The SQUIDs are connected to superconducting pick-up loops around the resonance cells via superconducting twisted wire pairs. The SQUIDs are read out in flux-locked loop mode using room temperature electronics and continuous digitalisation for uninterrupted tracking of the magnetic field. The resonance cell that is held neutral also acts as a neutron magnetometer complementing the SQUIDs.

\section{Conclusion}

We are currently in the commissioning phase of the experiment at the $\mathrm{H} 53$ beam position at ILL. We plan to make a measurement of the nEDM with sensitivity $10^{-27} e \mathrm{~cm}$ and move to a position of higher intensity $9 \AA$ neutron flux in 2012. At this position we will achieve a sensitivity of of the order $10^{-28} e \mathrm{~cm}$ with the sensitivity ultimately limited by systematic errors. This will be sufficient to discover the nEDM predicted by many "beyond the Standard Model" theories or to place a new upper limit on the nEDM, severely constraining theory.

\section{References}

[1] T. Ibrahim and P. Nath, APS 80 (2008) 577 [hep-ph/ 0705 . 2008v2]

[2] S. Dar, (2000) [hep-ph/0008248v2].

[3] J. Ellis, Nuclear Instruments and Methods in Physics Research Section A 284 (1989) 33

[4] C.A. Baker, D.D. Doyle, P. Geltenbort, K. Green, M.G.D. van der Grinten, P.G. Harris, P. Iaydjiev, S.N. Ivanov, D.J.R. May, J.M. Pendlebury, D.B. Shiers, K.F. Smith, Physical Review Letters 97 (2006) 131801.

[5] N.F. Ramsey, Phys.Rev. 78, 695 (1950).

[6] C.A. Baker, S.N. Balashov, J. Butterworth, P. Geltenbort, K. Green, M.G.D. van der Grinten, P.G. Harris, P. Iaydjiev, S.N. Ivanov, J.M. Pendlebury, D.B. Shiers, M.A.H. Tucker and H. Yoshiki, Physics Letters A $\mathbf{3 0 8}$ (2003) 67-74.

[7] H. Yoshiki, H. Nakai and E. Gutsmiedl, Cryogenics 45-6 (2005) pp.399-403

[8] C.A. Baker, S.N. Balashov, K. Green, M.G.D. van der Grinten, P.S. Iaydjiev, S.N. Ivanov, J.M. Pendlebury, D.B. Shiers, M.A.H. Tucker, H. Yoshiki and P. Geltenbort, Nuclear Instruments and Methods in Physics Research Section A 501 (2003) 517-523 